Document downloaded from:

http://hdl.handle.net/10251/159597

This paper must be cited as:

Castellanos, W.; Guerri Cebollada, JC.; Arce Vila, P. (2019). Available Bandwidth Estimation for Adaptive Video Streaming in Mobile Ad Hoc. International Journal of Wireless Information Networks. 26(3):218-229. https://doi.org/10.1007/s10776-019-00431-0

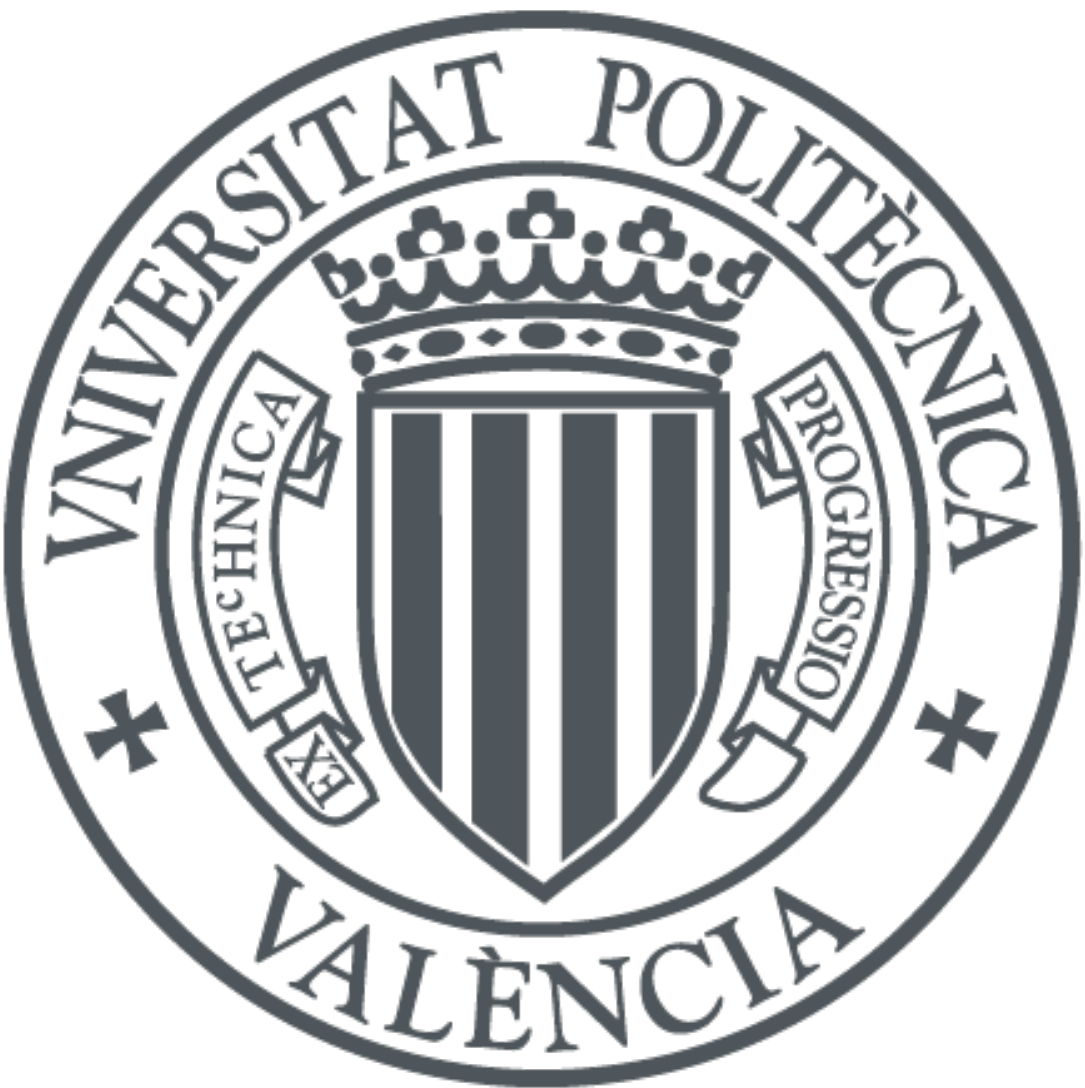

The final publication is available at

https://doi.org/10.1007/s10776-019-00431-0

Copyright Springer-Verlag

Additional Information 


\title{
Available bandwidth estimation for adaptive video streaming in mobile ad hoc
}

\author{
W. Castellanos, J.C. Guerri, and P. Arce
}

Institute of Telecommunications and Multimedia Applications (iTEAM), Universitat Politècnica de València, Spain, \{corresponding author: wilcashe@upv.es +573164837361; jcguerri@dcom.upv.es; paarvi@iteam.upv.es\}

\begin{abstract}
We propose in this paper an algorithm for available bandwidth estimation in mobile ad hoc networks and its integration into a conventional routing protocol like AODV for improving the rate-adaptive video streaming. We have introduced in our approach a local estimation of the available bandwidth as well as a prediction of the consumed bandwidth. This information allows video application to adjust its transmission rate avoiding network congestion. We conducted a performance evaluation of our solution through simulation experiments using two network scenarios. In the simulation study, transmission of video streams encoded with the H.264/MPEG-4 advanced video coding standard was evaluated. The results reveal performance improvements in terms of packet loss, delay and PSNR.
\end{abstract}

Keywords - Mobile ad hoc networks, available bandwidth estimation, video streaming, multi-bitrate video technique.

\section{INTRODUCTION}

A Mobile Ad hoc Network (MANET) is a type of wireless network where mobile nodes or terminals can communicate one another without any centralized administrator nor wired infrastructure. As the result of these infrastructure-less and easy-to-deploy characteristics, MANETs are very versatile and suitable to be used in many scenarios such as in battle zones and remote areas. Furthermore, recent advancements in the capabilities of mobile devices have led a rapid growth of the amount of multimedia traffic over MANETs. After several years of work on MANETs, this kind of networks are taking on new relevance due to they are very promising for many emerging scenarios and applications, including the networking platform for Internet of Things, VANETs (Vehicular ad-hoc networks) or future next-generation of $60 \mathrm{GHz}$ wireless 
communications [1]. Furthermore, recently a great interest has been focused on multimedia streaming over ah doc networks, as evidenced in references [2-7].

However, there are many challenges for providing satisfactory levels of quality-of-service (QoS) to end users, such as bandwidth-constrained, variable capacity links and energy-constrained operation. Additionally, as a consequence of the shared condition of the wireless channel as well as the dynamic network topology due to the mobility of the nodes, the transmission of time-sensitive data (e.g. video packets) is made more difficult [8]. Among multiple QoS parameters (e.g. delay, packet loss rate, and jitter) the available bandwidth is a very important parameter [9-11]. The estimation of the available bandwidth is one of the key strategies in order to improve the provision of network-adaptive video services over MANETs. However, an accurately estimation of the wireless link capacity is highly difficult as a consequence of the variable link conditions, which are caused by various factors such as mobility, fading, and interference. Although there are useful algorithms for available bandwidth estimation in wired networks, they assume the available bandwidth as the unused capacity of the tight link [12-14]. However, this is not an applicable concept to wireless ad hoc networks because of the unreliable and shared nature of the medium.

We propose in this paper an algorithm for available bandwidth estimation in MANETs and its integration into a conventional routing protocol like AODV for improving the rate-adaptive video streaming. Our algorithm introduces an estimation method performed in two phases: one for the estimation of the available bandwidth in each node and the other for the prediction of the consumed bandwidth along a route of $n$ hops taking into account the mutual interference.

In order to demonstrate the usefulness of the proposed solution we performed a simulation study. We have used in our experiments a video encoded according to the H.264/AVC standard. The performance evaluation was carried out using a modified version of the well-known Evalvid-RA framework [15], which integrates the network simulator NS-2 with external tools for analysing H.264/AVC video transmission. Evalvid-RA supports rate-adaptive multimedia transfer based on trace-file generation of an MPEG video file. The experimental results show that the combined use of our bandwidth estimation method and AODV provides an efficient strategy where a video application can adapt its traffic rate to the available bandwidth decreasing network congestion and obtaining a better video quality in terms of PSNR.

This paper is organized as follows. In Section 2 we provide an overview of the related works. Then, in Section 3 we briefly describe the impact of the share medium and the packet forwarding over the data rate in wireless ad hoc networks. Section 4 offers a more detailed explanation of our available bandwidth estimation method. Section 5 provides a description of the integration of our bandwidth estimation algorithm in AODV. In Section 6, we present the results of the performance evaluation of our approach and finally, we summarize our conclusions and discuss areas of future work in Section 7. 


\section{RELATED WORKS}

The shared nature of the wireless channel and interference between nodes make estimation of Available Bandwidth (BWav) in wireless ad hoc networks a challenging task. Few methods have been proposed so far to estimate the available bandwidth or the radio channel occupancy in ad hoc networks. In previous works, there are mainly three categories of approaches that could be adopted to the estimation of the available bandwidth in wireless ad hoc networks.

The first category comprises measurement-based algorithms. For instance, the algorithms based on Probe Rate Models (PRM) and Probe Gap Models (PGM). While the PRM algorithms use trains of probe packets at increasing rates for estimating the available bandwidth [16], the PGM algorithms bases the estimation on the dispersion gap between two consecutive probing packet. In [17] authors offer a survey of some approaches based on PRM and PGM. The main weakness of these approaches, especially when they are used in mobile ad hoc environment, is that they add high traffic overload.

A second category of approaches makes estimation of the available bandwidth using analytical models such as a Markov model [18, 19], an effective link model [20] or using Kalman filters [21, 22]. However, analytical models are highly topology-dependent and in a distributed and mobile scenario with a random topology, obtaining and maintaining the information required by an analytical model would be extremely difficult. For more details of the analytical techniques to improve the available bandwidth estimations, can be consulted the surveys published in $[14,23]$.

A third category consists of calculation-based approaches. For example, the solution presented in [24], measures certain performance metrics, for example, local information about the used bandwidth, in order to evaluate the available bandwidth. This local information is usually broadcast via HELLO messages, which are a type of packets used for discovering local topology in many routing protocols. If these exchanges are not too frequent, this technique can be reasonably considered as non-intrusive. Other approaches $[25,26]$ use MAC layer information to determine available bandwidth and delay information. These approaches are based on the bandwidth estimation of a link in discrete time intervals by averaging the throughputs of the recent packets in the past time window and use it to estimate the bandwidth in the current time window. Obviously, this estimation may not be accurate because the channel condition may have changed. In [9] authors suggest a distributed estimation of the available bandwidth with the method of channel monitoring, collision estimating and backoff duration predicting. Nevertheless, as a result of the random appearance of the collision and backoff procedure, the channel monitoring cannot reflect the future status on the link. These shortcomings were improved in [27]. In this work, authors proposed a scheme for estimating available bandwidth in MANETs based upon collision probability, idle period synchronization and random waiting time. In this approach, the collision probability at each node is estimated using distributed Lagrange Interpolation polynomial before the actual transmission of data. On the other hand, a frequent method for calculating the available bandwidth is based on estimating the minimum residual bandwidth among the intermediate nodes throughout the route [28, 
29]. Nonetheless, this method is fundamentally inaccurate since the fact of measuring the utilization of the medium locally causes the self-interference of a flow at consecutive links and the simultaneous idle times of neighbour links to be ignored.

\section{BACKGROUND}

One of the limitations of wireless ad hoc networks is the achievable capacity due to the fact that nodes cannot simultaneously access the shared medium. More specifically, when a node is transmitting a packet, neighbour nodes within its Interference Range (IR), have to keep silent. This fact degrades the wireless data rate. Even more, when a transmission is established, the nodes must cooperate to forward the packets through the network, which means that the available throughput on each host is limited not only by the access channel, but also by the forwarding load.

The results of the study published in [30] suggest that capacity along a route can be surprisingly low. As the number of hops increases, the maximum throughput of one flow substantially decreased due to the overhead of MAC layer and the mutual interference between packets of the same flow, also called "Intra-flow contention" [31]. Intra-flow contention occurs when nodes along a multihop route contend among themselves for channel access to forward packets belonging to the same flow. An example of the variation of the link capacity is shown in Figure 1. In this example, the wireless network has IEEE 802.11 as MAC protocol and 2 Mbps of channel capacity.

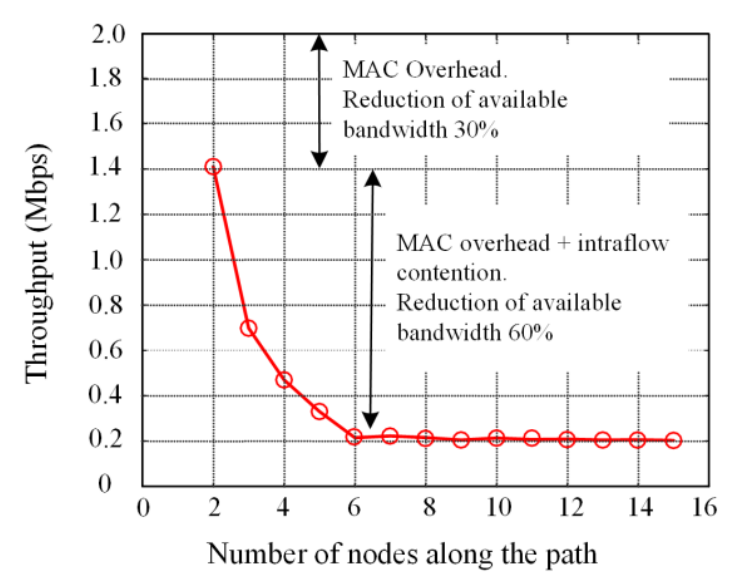

Fig. 1 Wireless link capacity as a function of the number of nodes along a route

\section{BANDWIDTH ESTIMATION ALGORITHMS}

Our bandwidth estimation solution is based on a scheme of two different stages. In the first stage, each node calculates its available bandwidth ( $B W a v$ ), using only its local perception, in order to know the available endto-end bandwidth along the route. We assume that the available bandwidth in a node is defined as the maximum throughput that can be achieved by a node without disrupting any ongoing flow in the network. This 
assumption is used in other works, such as [26] and [32]. However, not only the locally available resources must to be considered when analysing the required bandwidth for an incoming flow. Therefore, in the second stage we use an algorithm to predict the total amount of bandwidth that the new flow can consume $\left(B W_{\text {consumed }}\right)$ along a route of $n$ hops taking into account the Intra-flow contention. We cannot put this kind of potential interference into consideration while estimating the locally available bandwidth. Therefore, only is possible estimate it when a new flow starts the route discovery process.

In next subsections, we provide a brief description about the algorithms used to estimate the Local Available Bandwidth $\left(B W_{a v}\right)$ in each node and to predict the bandwidth to be consumed ( $\left.B W_{\text {consumed }}\right)$ by the requesting flow.

\section{A. Estimation of local available bandwidth}

Our proposed algorithm for estimating local available bandwidth consists of two steps. In the first step, each node estimates its local available bandwidth and, in the second one, the nodes calculate a weighted average of the most recent values in order to obtain a final estimation of the local available bandwidth.

In the first step, to estimate the local available bandwidth (remaining bandwidth) between two neighbour nodes we assume the following definition:

Definition 1: The local available bandwidth between two nodes is defined as the maximum throughput that can be transmitted between these two nodes without negatively affecting any ongoing flow in the network (permissible throughput).

The Definition 1 should not be confused with the classical definition that considers the available bandwidth as the unused capacity of the link. The problem of the estimation of the local available bandwidth can be, therefore, re-formulated as the determination of the maximum throughput that can be used for the data transmission between two nodes in the current time. This assumption is used in other works [24], [26] and $[33]$.

In our approach, a given node can estimate its permissible throughput to each neighbour by the Equation 1 . Where $S$ is the size (in terms of bits) of the packet sent from the node to its neighbour. $T s$ is the time-stamp when the packet is ready at the MAC layer, and $\operatorname{Tr}$ is the time-stamp when an ACK has been received (see Figure 2).

$$
T h_{\text {packet }}=\frac{S}{T r-T s}
$$




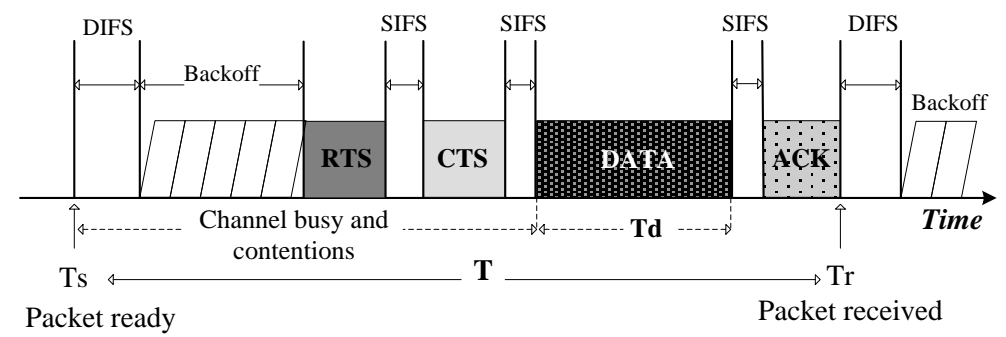

Fig. 2 Transmission of a single packet in IEEE 802.11 DCF MAC protocol

Several methods have been proposed in order to measure the parameters of Equation 1. For example, In [28], authors propose to use a train of probe packets in order to observe the network performance. However, this technique introduces additional traffic into the network. Another method was used in [34] where parameters $T s, T r$ and $S$ were obtained by modifying the MAC layer. However, it requires that changes must be performed to the firmware of standard 802.11 hardware. We propose a different mechanism in the measurement of the parameters of the Equation 1. This allows for a more accurate estimation of the local available bandwidth as well as an implementation easier. In our approach, each node in the network can estimate the permissible throughput to its neighbours by using existing HELLO packets, which are used to discover neighbours in AODV. Our algorithm has two main advantages. First, it can be implemented in network layer, as opposed to firmware. Second, more overload to the network is avoided due to the use of the HELLO packets of AODV.

The implementation of our algorithm can be explained with more details as follows. Let $i$ be a node and $j$ its neighbour. To estimate the permissible throughput at the node $i$, a HELLO packet is sent from node $i$ to $j$. The time-stamp (Ts) when the packet is ready to be sent is recorded. When node $j$ receives the HELLO packet, it sends back to node $i$ a HELLOAck packet carrying the time-stamp $T s$. Finally, the time-stamp $T r$ is recorded in node $i$ when the HELLOAck packet is received. HELLOAck packet is an additional packet created in our implementation which allows the available bandwidth calculation to be more reliable since it does not depend of the throughput seen by only one packet. Our available bandwidth estimation, based on the exchange of HELLO and HELLOAck packets, includes the channel busy, the contention time, the MAC overhead and the current time used for data transmission. Therefore, in order to understand what our algorithm includes, the Equation 1 can be rewritten using the explicit form shown in Equation 2. This equation is expressed in terms of $B W a v$ according to the Definition 1.

$$
\begin{aligned}
& B W_{a v} \\
& =\frac{R T S+C T S+\text { Hello }+ \text { ACK }+ \text { RTS }+ \text { CTS }+ \text { HelloAck }}{T r-T S}
\end{aligned}
$$

Where,

$$
\operatorname{Tr}-T s=T_{Q}+\left(T_{\text {Hello }}+T_{\text {Hello }}+T_{C P}+T_{C P}\right) \times R+\sum_{1}^{R} B_{T}
$$


According to the Equation 2, the packets sent and received during the time interval $T r$ - $T s$ involve not only the HELLO packets, but also the MAC control packets (RTS, CTS and ACK). The period of time Tr-Ts takes into account the time used for transmitting HELLO and HELLOAck packets $\left(T_{\text {Hello }}, T_{\text {HelloAck }}\right)$, the queuing time $T_{Q}$, collision avoidance phase time (SIFS, DIFS) $T_{C A}$, the control overhead time (RTS, CTS) $T_{O H}$ and back-off time $B_{T}$ for $R$ retransmissions.

Our measurement mechanism captures the effect of channel contention, fading and interference errors. Since, if contention is high, $T r-T s$ will increase, and therefore, the available bandwidth will decrease. This mechanism also captures the effect of physical errors because if the RTS or HELLO packets are affected by channel errors, they have to be re-transmitted. Thus, the interval $T r$ - $T s$ is increased and correspondingly the available bandwidth perceived by the node is decreased.

The rate of the estimation of the local available bandwidth at each node is the interval of time $\Delta t$, which corresponds to the period between HELLO messages. The accuracy of the bandwidth calculation depends on the value of $\Delta t$. A small interval increases the precision of the estimation but increases the overhead. Therefore, there is a trade off between the duration of the interval and the bandwidth usage. However, $\Delta t$ should be small enough to allow fast reactions to change of the load and the topology. In addition, the periodic nature of the HELLO generation has the disadvantage that it increases the risk of simultaneous transmissions resulting in collisions. Therefore, we choose as HELLO interval $(\Delta t)$ a random value between 1 and 1.5 seconds, which is the recommended value in [35]. This decreases the probability of simultaneous transmissions.

In the second step of the estimation of the local available bandwidth, each node calculates a weighted average of the most recent values of the Local Available Bandwidth in order to obtain a stable and yet responsive system of estimation (Equation 4).

$$
\widehat{B W}_{a v}\left(t_{i}\right)= \begin{cases}\alpha B W_{a v}\left(t_{i}\right)+(1-\alpha) \times \widehat{B W}_{a v}\left(t_{i}-1\right), & t>0 \\ B W_{a v}\left(t_{o}\right), & t=0\end{cases}
$$

Where $\widehat{B W}_{a v}\left(t_{I}\right)$ is the new weighted average of the local available bandwidth, $B W_{a v}\left(t_{I}\right)$ is the actual measurement of the local available bandwidth in the time period $t_{i}, \widehat{B W}_{a v}\left(t_{i-1}\right)$ is the weighted average calculated in the previous time period $t_{i}-1$, and $B W_{a v}\left(t_{o}\right)$ represents the initial measurement of the local available bandwidth. We use $\alpha=0.8$, which were determined by a few empirical trials. This value of $\alpha$ allows the algorithm a fast reaction to changes of the network condition.

In summary, in order to estimate the local available bandwidth in a given node we calculate the permissible throughput between this node and its neighbours in the current time, using exchange of HELLO packets. The measured throughput allows the node to infer the value of the available bandwidth at that node. Finally, in order to obtain a stable and yet responsive system of estimation, each node calculates a weighted average of the Local Available Bandwidth $\left(\widehat{B W}_{a v}\right)$. Results show that this method is the most conservative of all the 
methods discussed and it gives the more accurate results. Figure 3 illustrates how to use the HELLO and HELLOAck packets in the estimation of the permissible throughput.

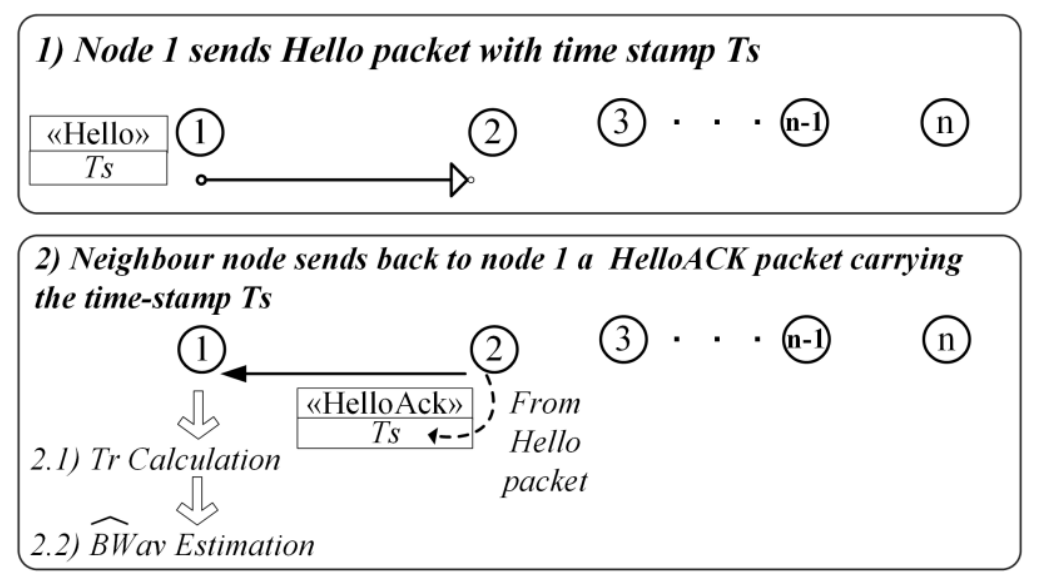

Fig. 3 Bandwidth estimation using the Hello packets

\section{B. Prediction of the consumed bandwidth along the path}

The second stage of the bandwidth estimation method consists of estimating the consumed bandwidth along the path to check if all nodes along the route could support the bandwidth requested by the source. In order to calculate this consumed bandwidth, it is necessary to take into account the mutual interference between packets of the same flow. This sub-phase is executed only by the destination node when a new flow starts the route discovery process.

Our method for calculating the intra-flow contention is based on the parameter called Contention Count $(C C)$. The impact of the mutual contention between nodes has been studied in some works, for example in [36, 37]. The value of this parameter will help to calculate the consumed bandwidth for the route during a transmission. In our method, the interference range is assumed to be more than twice of the transmission range. It means that the nodes that are one hop or two hops away from the transmitter will get the interference and cannot use the channel. In other words, this method only considers the interference coming from nodes that are one hop and two hops away.

The contention count is calculated as shown Equation $5[36,37]$.

$$
\mathrm{CC}=h_{r e q}+h_{r e p}
$$

The calculation of the $h_{r e q}$ and $h_{\text {rep }}$ parameters must satisfy the next two conditions:

$$
\begin{aligned}
& \text { i) if } h_{\text {req }}>2 \text { then } h_{\text {req }}=2 \\
& \text { ii) if } h_{\text {rep }}>3 \text { then } h_{\text {rep }}=3
\end{aligned}
$$


The $h_{r e q}$ and $h_{r e p}$ parameters mean the number of hops from one node to the source and destination nodes, respectively. They can be directly obtained from the Hop Count field of the RREQ and RREP packets of AODV.

Figure 4 shows an example in order to explain the calculation of $C C$ in a clear way. In the example, we assume that there is a flow intended to be sent from node 1 to node 6 . Taking the node $3, h_{\text {req }}$ is 2 and $h_{\text {rep }}$ is 3 , then $C C=2+3=5$ according to the Equation 5. Figure 4 shows how each node calculates its $C C$ parameter. Irn means that the node is in the interference range of node $n$ that is transmitting. Similarly, Trn means that the node is in the interference range of node $n$ that is transmitting. For example, when node 3 is transmitting packets to node 4, node 1, 2 and 5 will get the interference and the channel for these nodes should be set as busy since they share channel with node 3 . We show how the maximum $C C$ value is obtained in the node 3 , which causes a bandwidth bottleneck at that node. In Figure 4 we can see that for a route with 5 or more hops the CCmax has a fixed value of 5 and for routes with less than 5 hops, CCmax is equal to the number of hops.

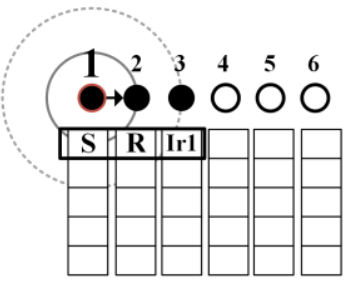

(a)

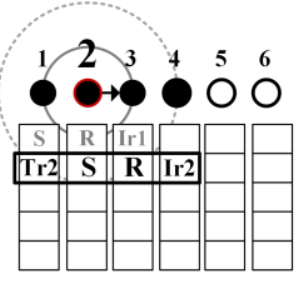

(b)

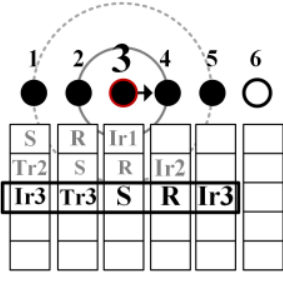

(c)

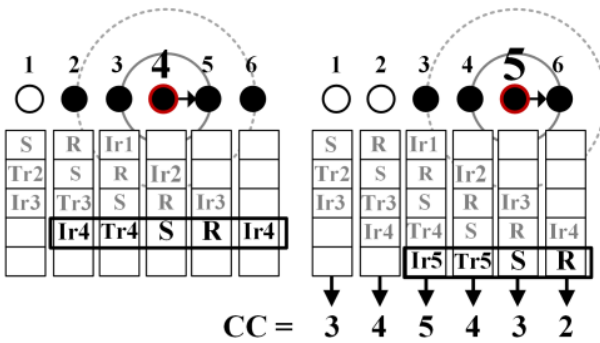

(e)

$I r_{n}$ : node is in the Interference Range of node $n, T r_{n}$ : node is in the Transmission Range of node n, CC: Contention Count, S: Sender, R: Receiver

Fig. 4 Example of Intra-flow contention calculation

After estimating CCmax, destination node could calculate the consumed bandwidth as shown in the Equation 6. Where reqBW is the bandwidth requested by the source node, CCmax indicates the maximum value of the contention count in the route and $B W_{\text {consumed }}$ means the consumed bandwidth expected along the route if a flow is transmitted through path with data rate equal to $r e q B W$.

$$
B W_{\text {consumed }}=C C \text { max } \times \text { reqBW }
$$

In next section, we explain how our bandwidth estimation algorithm can be used for improving the route discovery process of a routing protocol like AODV.

\section{ENHANCED ROUTE DISCOVERY BASED ON ESTIMATION OF THE AVAILABLE BANDWIDTH}

The route discovery process is executed when a source node requested a route to a destination node with specific bandwidth requirements. Therefore, it broadcasts a $R R E Q$ packet to its neighbour nodes. When a node receives a $R R E Q$ packet, it is rebroadcasted. This process continues until the $R R E Q$ packet reaches the 
destination node. In AODV, a RREP packet can be created by the destination node or an intermediate node with a "fresh enough" route to the destination. However, only the destination will be able to send the route reply packet $(R R E P)$ in our approach. This will ensure that all nodes in the selected route satisfy the bandwidth constraints. When the destination node receives a $R R E Q$ packet, it calculates the $C C$ max and the $B W_{\text {consumed }}$ according to the Equation 5 and Equation 6 respectively. Subsequently, the destination node compares the $B W_{\text {consumed }}$ with the last value of its local available bandwidth $\left(\widehat{B W}_{a v}\right)$, which has been calculated using the Equation 4. If $B W_{\text {consumed }}$ is less than $\widehat{B W}_{a v}$ in the destination node, it informs the source node that the transmission rate must be equal to the requested bandwidth (reqBW). Otherwise, if the $\widehat{B W}_{a v}$ in the destination node is less than the value of $B W_{\text {consumed }}$, the source must adjust its transmission rate to $\widehat{B W}_{a v} / \mathrm{CC}_{\max }$. Finally, the RREP will be transmitted to the source with a modified header that includes the minimum value between requested bandwidth by the source $(r e q B W)$ and the maximum bandwidth that all nodes along the route could support taking into account the intra-flow contention, (i.e. $\widehat{B W}_{a v} / C C_{\max }$ ). Once an intermediate node receives the RREP packet, it compares its local available bandwidth with the bandwidth indicated in the RREP. If its local available bandwidth is lower, it updates the min-bandwidth field in $R R E P$, using its available bandwidth. Otherwise, the node forwards the RREP. This procedure will ensure that the source knows the minimum bandwidth along the path, which will be the maximum rate that it may transmit. As an example, let us consider the wireless ad hoc network described in Figure 5, where the available bandwidth (in Kbps) of each link is known. We assume that the source node requests to send data with a rate of $120 \mathrm{Kbps}$ (reqBW) to node 6 . In this case, the CCmax calculated will be 5 and therefore the value of $B W_{\text {consumed }}$ will be $600 \mathrm{Kbps}$ (i.e. $120 \mathrm{Kbps} \times 5)$ according to Equation 6. Assuming that the local available bandwidth $\left(\widehat{B W}_{a v}\right)$ in the node 6 is $400 \mathrm{Kbps}$, we can see that the consumed bandwidth ( $\left.B W_{\text {consumed }}\right)$ is higher than the $\widehat{B W}_{a v}$.

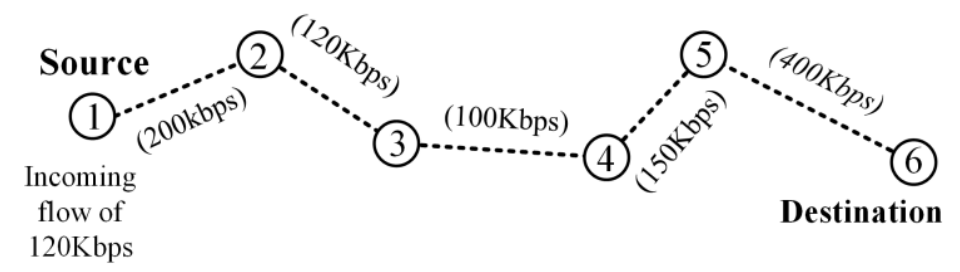

Fig. 5 Example of wireless ad hoc network with the available bandwidth of each link

This means that if the source node transmits to $120 \mathrm{Kbps}$ the destination could not support such rate. Therefore, the destination calculates a new rate according to its local available bandwidth. Then the destination node notifies the source that the transmission rate must be $80 \mathrm{Kbps}\left(\widehat{B W}_{a v} / C C_{\max }=400 \mathrm{Kbps} / 5\right)$.

\section{SIMULATIONS AND PERFORMANCE EVALUATION}

In this section, we evaluate the performance of our approach and compare it with the well-known AODV protocol, which does not include bandwidth estimation algorithms. We plot the performance of AODV in the graphs in order to emphasize the performance improvements regarding the typical routing protocols. In 
addition, the bandwidth estimation algorithm included in the QAODV protocol [29] is evaluated. We take QAODV for performance comparison with our approach, because it is the closest protocol to our modified AODV version.

The objective of our simulation study is twofold. First, to evaluate the performance of our approach. And second, to demonstrate the effectiveness of our proposed protocol in a video transmission over MANETs.

\section{A. The Simulation Environment}

Network Simulator 2 (NS-2) has been used to test the performance of our solution. NS-2 contains the IEEE802.11 protocol in the MAC layer working in the distributed coordination function (DCF) mode with a channel data rate of 2 Mbps. The radio propagation model is Two Ray Ground and queue type is Drop Tail with maximum length of 50 packets. The transmission range and interference range are $250 \mathrm{~m}$ and $550 \mathrm{~m}$ respectively.

In order to simulate H264/AVC video transmission using NS-2, we have used a modified version of the Evalvid-RA framework [15], which is well-accepted for evaluation of the quality of video transmitted over a real or simulated communication network. Evalvid-RA supports rate-adaptive multimedia transfer based on trace-file generation of an MPEG video file. The traffic flow used in our simulations consists of a video stream, which has been created by concatenating some well-known test sequences such as mobile, paris, foreman and akiyo [38] (See Figure 6). The resulting video has 3607 frames and it is encoded at 15 frames per second (fps) with a resolution of 352x288 pixels (CIF, Common Intermediate Format).

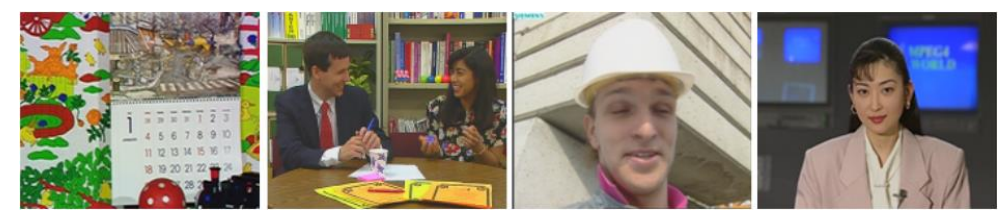

Fig. 6 Screenshots of the test sequences used in the simulations

As shown in Figure 7, we generated ten different versions of the original video sequence each with different bitrates (from $100 \mathrm{Kbps}$ to $1 \mathrm{Mbps}$ ). The ten video streams were generated using different encoding parameters, this process is known as multi-bitrate video technique. 


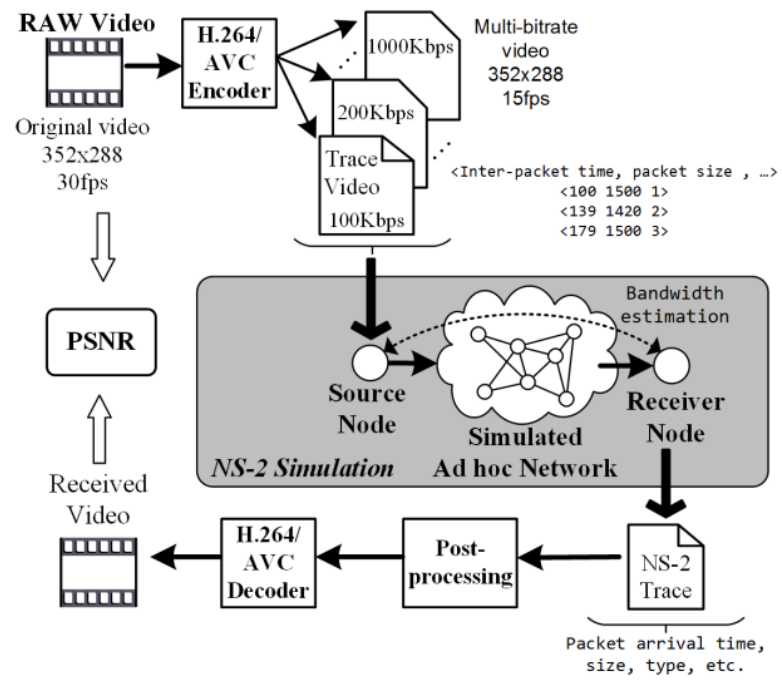

Fig. 7 Simulation methodology: multi bit-rate video technique using H.264/AVC

Our simulation methodology allows source node to keep multiple bitrate versions of the video sequence stream the content to a destination node with a bit rate that closely matches the available bandwidth estimated by our algorithm (see Figure 7). After simulation, received video was used to calculate the PSNR (Peak Signal-to-Noise Ratio) for each frame.

In addition to the video flow, five CBR (Constant Bit Rate) flows over UDP of $10 \mathrm{Kbps}$ each were introduced randomly as background traffic in the network. In order to evaluate the quality of the transmitted video we have done several measurements and calculations (involving network and video metrics) such as throughput, packet delivery ratio, delay and PSNR, which are parameters related to the objective quality of the reconstructed videos.

We have used two simulation scenarios. The first scenario is used to provide a clear exposition of the operation of solution. The second scenario is a more realistic scenario of 30 mobile nodes. For each type of scenario, we run the simulation for 15 times (with random scenarios with different seeds) to take average values in the measured performance metrics. The results are obtained with a confidence level of $95 \%$. In figures, our approach is denoted as AODV with Bandwidth Estimation (BWE+AODV).

\section{B. Simulation results}

1) Scenario 1: static linear topology with variable length

The first scenario is illustrated in Figure 8 and consists of a variable number of nodes placed in a linear topology in order to observe the performance of the protocol in a deterministic environment. 


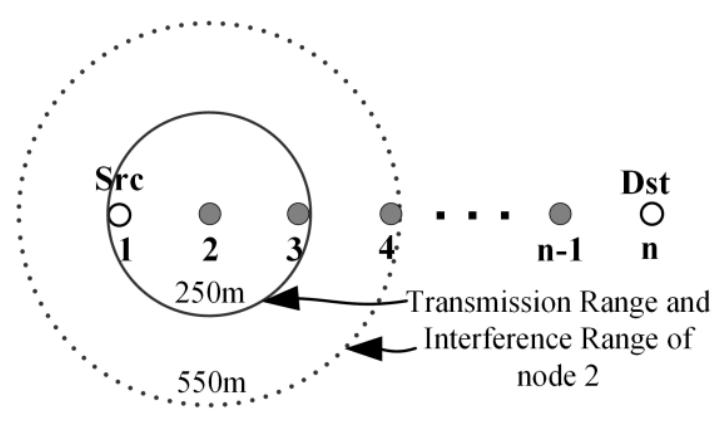

Fig. 8 First scenario: static linear topology with variable length. The transmission and interference range for node 2 are shown

Node 1 is the video source and the last node in the chain is the traffic sink. In this scenario, the performance of our solution was tested as function of the number of hops on the path. Initially the source requested a transmission rate of $0.35 \mathrm{Mbps}$, which be maintained constant when AODV and QAODV are used but can be changed by the source when our approach is used because of the adaptive feedback scheme.

As observed in Fig. 9, total network throughput obtained with our approach is very close to throughput achieved with AODV. However, using AODV the source does not know the available bandwidth and injects packets to the network with data rate equal to the requested rate $(0.35 \mathrm{Mbps})$ by the video application. This transmission rate can be efficiently supported by routes composed of up to three nodes, but not by routes consisting of more than three nodes. As a consequence of this fact, data packets cannot be efficiently forwarded along the path and, therefore, the network congestion and packet loss are increased significantly. On the other hand, when our solution is used in the network, source node adjusts its data rate to the maximum rate that the route can support. This implies that in routes with several hops, the transmission rate is lower than the one configured by the source when AODV is used. Therefore, the packet loss (Fig. 10) and delay (Fig. 11) are significantly reduced without sacrificing the throughput achieved in the destination node. This fact provides better conditions to video streaming applications as discussed later.

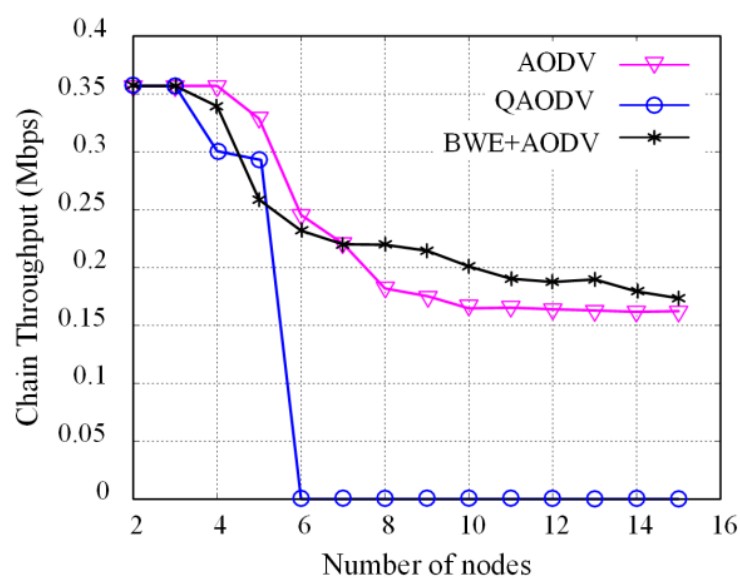

Fig. 9 Throughput achieved along the path as a function of the route length 


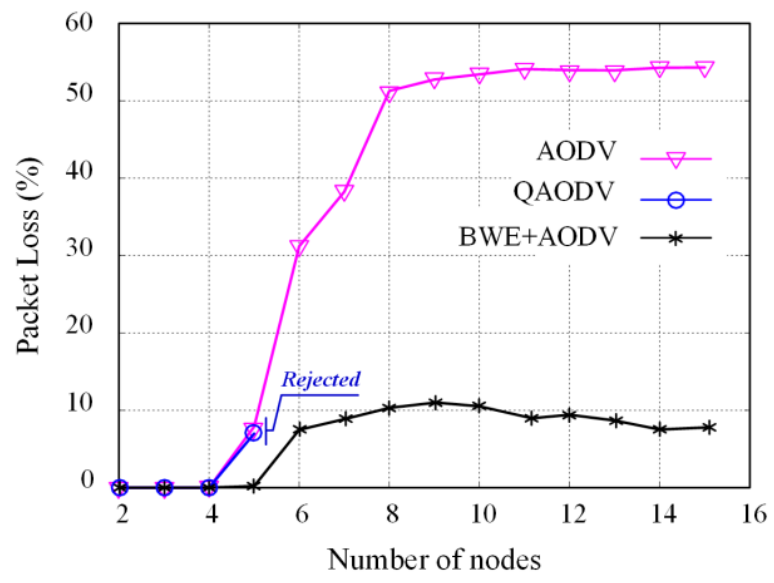

Fig. 10 Packet Loss as a function of the route length

Regarding QAODV, its admission control system only accepts the traffic flow of $0.35 \mathrm{Mbps}$ when the number of hops between source and destination is less than 4. In these cases, QAODV presents a similar behavior to AODV in terms of throughput, delay and packet loss. In the figures, we add the label "rejected" on the curves of QAODV in order to denote that the traffic flow is rejected by the admission control system when the route cannot support the requirements of the flow.

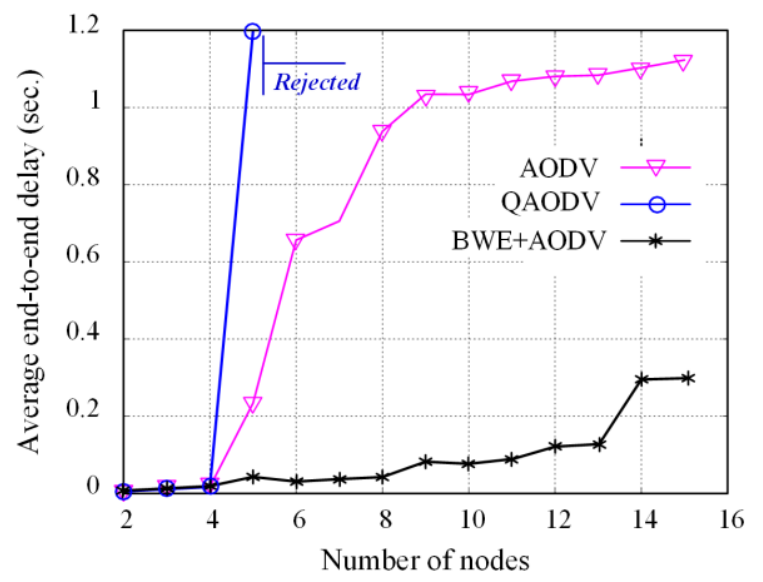

Fig. 11 Average end-to-end as a function of the route length

Concerning video transmission, Figure 12 shows the average PSNR obtained during the network simulation. Initially, the three protocols send video packets to a same rate of $0.35 \mathrm{Mbps}$. This transmission rate can be supported by a route of up to 4 nodes. Therefore, the PSNR obtained by the three approaches is very similar during this simulation segment. However, for routes with five or more nodes, the data rate of $0.35 \mathrm{Mbps}$ cannot supported by network. As available bandwidth decreases, due to the number of nodes increases, the PSNR values decrease for the three protocols. In the case of our approach, this reduction is mainly caused by bit-rate adaptation performed by the video application, which sends video packets a lower bit rate. 
Figure 12 clearly shows that our approach provides the highest video quality. When the route has five nodes, our solution sends video packets to a rate of $0.3 \mathrm{Mbps}$ and the average PSNR obtained is of $30 \mathrm{~dB}$ (an improvement of $7 \mathrm{~dB}$ and $4 \mathrm{~dB}$ in relation with AODV and QAODV, respectively). When the route has six hops o more, the available bandwidth remains constant and the quality of the received video is improved in 11 $\mathrm{dB}$ in relation with AODV.

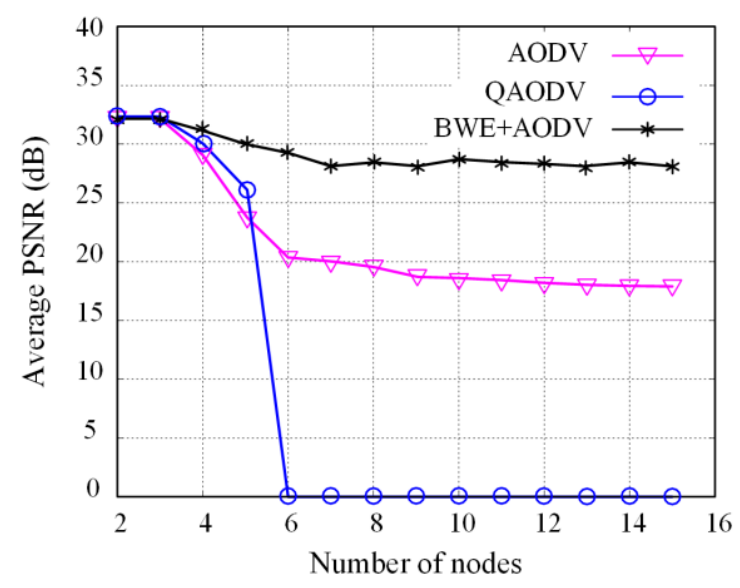

Fig. 12 Average PSNR as a function of the route length

\section{2) Scenario 2: mobile topology}

In this simulation scenario, 30 nodes move in a $1000 \mathrm{~m} \times 1000 \mathrm{~m}$ area according to the random waypoint model with pause time set to $5 \mathrm{~s}$. The nodes move toward a random destination using a speed between $0.1-2$ $\mathrm{m} / \mathrm{s}$. A random source-destination pair sends video packets using a request rate between 0.1 and $1.0 \mathrm{Mbps}$. In this network scenario, the transmission rate requested by source node is variable from 0.1 to $1 \mathrm{Mbps}$.

Figures 13, 14 and 15 show the results of our simulations in which the packet loss, average end-to-end delay and throughput are plotted versus the requested rate by source node. In figures, the evaluation results of our approach are denoted as BWE+AODV.

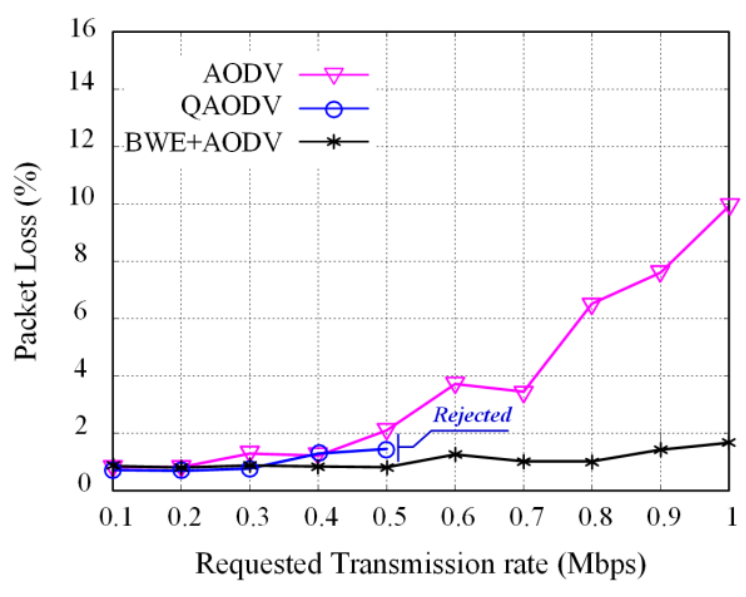

Fig. 13 Packet Loss with variable requested rate 


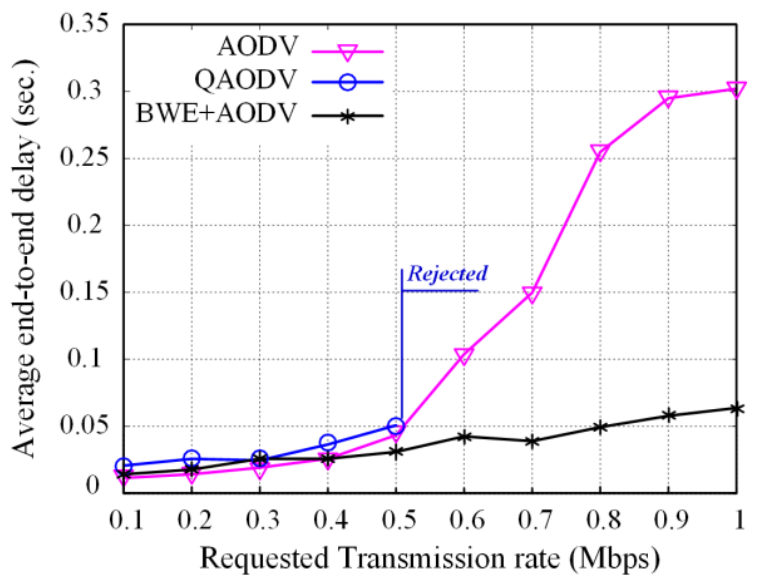

Fig. 14 Average end-to-end delay with variable requested rate

Figure 14 shows that the average end to end delay using our approach is always below $70 \mathrm{~ms}$, whereas, the end-to-end delay of AODV is significantly increased when the transmission rate increases from $500 \mathrm{Kbps}$ to $1000 \mathrm{Kbps}$. With AODV, the maximum average end-to-end delay reaches $300 \mathrm{~ms}$ at $1000 \mathrm{Kbps}$, about 4 times higher than using our solution. Although QAODV shows a delay lower than AODV, only can support traffic flows below $0.5 \mathrm{Mbps}$ in this scenario.

As seen in Figure 15 the total network throughput achieved with BWE+AODV is very close to throughput achieved using AODV. Although there is a degradation of the BWE+AODV performance in scenarios with high mobility (compared with static scenarios) since the nodes need a higher time for exchanging information about the network status, our proposal still shows better performance than the other alternatives.

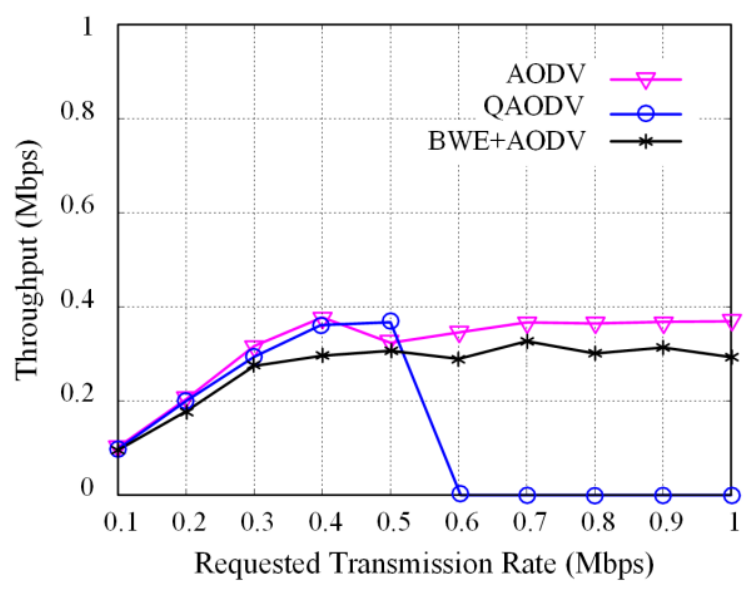

Fig. 15 Throughput with variable requested rate

Figure 16 shows how the average PSNR drastically diminishes (using AODV) when the route cannot support efficiently the data rate requested by the source. On the contrary, using our proposal the application can appropriately adjust its transmission rate according to bandwidth estimation. Therefore, the quality of the video is not degraded compared to the achieved level with the maximum rate that the path can provide $(0.5 \mathrm{Mbps})$. 
As seen in Figure 16, to values above $0.5 \mathrm{Mbps}$, the PSNR is very close to $30 \mathrm{~dB}$ in the case of BWE+AODV while using AODV it decreases to reach $18 \mathrm{~dB}$ (about $12 \mathrm{~dB}$ of difference) in the worst case. In general terms, we can say that the effective estimation of the available bandwidth of BWE-AODV helps avoid or reduce the congestion in MANETs, which provide better conditions to video streaming applications.

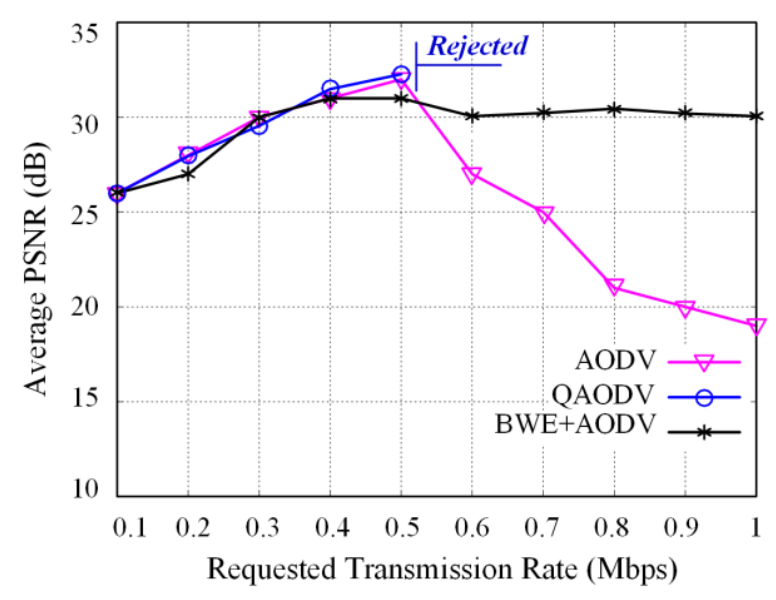

Fig. 16 Average PSNR with variable requested rate

\section{CONCLUSIONS}

An algorithm for estimating the available bandwidth in MANETs is proposed in this paper. Our algorithm includes a local estimation of the available bandwidth in each node of the network as well as a prediction of the bandwidth consumed by a data flow when it is transmitted through a path. We have integrated our estimation algorithm in the AODV routing protocol in order to improve the rate-adaptive video streaming over mobile ad hoc networks. This integration provides a suitable system for supporting an adaptive strategy where video source can adapt its data rate avoiding network congestion and achieving a significantly improvement in the quality of the transmitted video.

Moreover, we conducted a performance evaluation of our proposal with other routing protocols, like AODV and QAODV. Experimental results show that our solution can avoid network congestion and provide an efficient solution adaptive video transmission over MANETs.

In terms of video transmission, the obtained results prove that our solution makes a more efficient use of the available bandwidth since it provides feedback to the traffic source about the available bandwidth to appropriately adjust the transmission rate. When this adaptive procedure is not performed, the packet delay is significantly increased and a large percentage of packets are dropped, which is much worse than sending video with a lower bit rate.

As future works, we plan to perform experiments using bandwidth estimation algorithm and different adaptive streaming techniques. Particularly, our future work includes the development of a video evaluation framework that integrates available bandwidth estimation with an adaptive rate controller using Scalable High 
Efficiency Video Coding (H.265/SHVC) [39] and the MPEG-DASH (Dynamic Adaptive Streaming over HTTP) [40] technique.

\section{REFERENCES}

1. Khorov, E., Krasilov, A., Krotov, A., Lyakhov, A.: Will MCCA revive wireless multihop networks? Comput. Commun. 104, 159-174 (2017). https://doi.org/10.1016/j.comcom.2016.10.004.

2. Immich, R., Cerqueira, E., Curado, M.: Efficient high-resolution video delivery over VANETs. Wirel. Netw. 1-16 (2018). https://doi.org/10.1007/s11276-018-1687-2.

3. Usman, M., Jan, M.A., He, X., Alam, M.: Performance evaluation of High Definition video streaming over Mobile Ad Hoc Networks. Signal Process. 148, 303-313 (2018). https://doi.org/10.1016/j.sigpro.2018.02.030.

4. Liu, J., Yuan, X., Qian, H., Cheng, Y., Liu, F.: Research on video transmission Ad Hoc network routing technology oriented multimedia applications. J. Intell. Fuzzy Syst. 34, 879-886 (2018). https://doi.org/10.3233/JIFS-169381.

5. Castellanos, W., Guerri, J.C., Arce, P.: Performance Evaluation of Scalable Video Streaming in Mobile Ad hoc Networks. IEEE Lat. Am. Trans. 14, 122-129 (2016). https://doi.org/10.1109/TLA.2016.7430071.

6. Sharma, L., Lal, C., Sharma, D.P., Kaliyar, P.: Enhancing QoS for Multimedia Services Using MobilityAware Bandwidth Estimation Algorithm in MANETs. In: Optical and Wireless Technologies. pp. 655666. Springer, Singapore (2018). https://doi.org/10.1007/978-981-10-7395-3_73.

7. Castellanos Hernandez, W.: Quality of Service Routing and Mechanisms for Improving Video Streaming over Mobile Wireless Ad hoc Networks, https://riunet.upv.es/bitstream/handle/10251/53238/CASTELLANOS\%20\%20Quality\%20of\%20Service\%20Routing\%20and\%20Mechanisms\%20for\%20Improving $\% 20$ Video\% 20Streaming\%20over\%20Mobile....pdf?cv=1\&isAllowed=y\&sequence=10, (2015).

8. Basarkod, P.I., Manvi, S.S.: On-demand Bandwidth and Stability Based Unicast Routing in Mobile Adhoc Networks. Int. J. Electron. Telecommun. 60, 20-32 (2014).

9. Peng, Y., Yan, Z.: Available Bandwidth Estimating Method in IEEE802.11e based Mobile Ad Hoc Network. In: 9th International Conference on Fuzzy Systems and Knowledge Discovery (FSKD). pp. 2138-2142. , Chongqing (2012). https://doi.org/10.1109/FSKD.2012.6234276.

10. Salcedo, D., Guerrero, J., Guerrero, C.D.: Overhead in Available Bandwidth Estimation Tools: Evaluation and Analysis. Int. J. Commun. Netw. Inf. Secur. 9, 393-404 (2017).

11. Zhen, X., Wenzhong, Y.: Bandwidth-aware routing for TDMA-based mobile ad hoc networks. Int. Conf. Inf. Netw. ICOIN. 637-642 (2013). https://doi.org/10.1109/ICOIN.2013.6496701.

12. Salcedo, D., Guerrero, C., Martínez, R.: Available Bandwidth Estimation Tools Metrics, Approaches and Performance. Int. J. Commun. Netw. Inf. Secur. IJCNIS. 10, (2018).

13. Airon, M., Gupta, N.: Bandwidth Estimation Tools and Techniques: A Review. (2017). https://doi.org/10.20944/preprints201710.0060.v1.

14. Chaudhari, S.S., Biradar, R.C.: Survey of Bandwidth Estimation Techniques in Communication Networks. Wirel. Pers. Commun. 83, 1-52 (2015). https://doi.org/10.1007/s11277-015-2459-2.

15. Lie, A., Klaue, J.: Evalvid-RA: trace driven simulation of rate adaptive MPEG-4 VBR video. Multimed. Syst. J. 14, 33-50 (2008). https://doi.org/10.1007/s00530-007-0110-0.

16. Paul, A.K., Tachibana, A., Hasegawa, T.: An Enhanced Available Bandwidth Estimation Technique for an End-to-End Network Path. IEEE Trans. Netw. Serv. Manag. 13, 768-781 (2016). https://doi.org/10.1109/TNSM.2016.2572212.

17. Farshad, A., Lee, M., Marina, M.K., Garcia, F.: On the impact of 802.11n frame aggregation on end-toend available bandwidth estimation. In: Eleventh Annual IEEE International Conference on Sensing, Communication, and Networking (SECON). pp. 108-116. , Singapore (2014). https://doi.org/10.1109/SAHCN.2014.6990333.

18. Liao, X., Yang, Z., Yuan, P.: An unscented Kalman filter based available bandwidth estimation algorithm for space bundle links. In: 9th International Conference on Wireless Communications and Signal Processing (WCSP). pp. 1-6 (2017). https://doi.org/10.1109/WCSP.2017.8171064. 
19. Suganya, R., Jayashree, L.S.: Fuzzy Rough Set Inspired Rate Adaptation and resource allocation using Hidden Markov Model (FRSIRA-HMM) in mobile ad hoc networks. Clust. Comput. 1-14 (2018). https://doi.org/10.1007/s10586-018-1783-1.

20. Avestimehr, A.S., Diggavi, S.N., Tse, D.N.C.: Wireless Network Information Flow: A Deterministic Approach. IEEE Trans. Inf. Theory. 57, 1872-1905 (2011). https://doi.org/10.1109/TIT.2011.2110110.

21. Zhang, X., Nguyen, T.M.T., Pujolle, G.: Kalman filter based bandwidth estimation and predictive flow distribution for concurrent multipath transfer in wireless networks. In: 3rd IEEE International Conference on Network Infrastructure and Digital Content (IC-NIDC). pp. 305-309. , Beijing (2012). https://doi.org/10.1109/ICNIDC.2012.6418765.

22. Houssaini, Z.S., Zaimi, I., Drissi, M., Oumsis, M., Ouatik, S.E.A.: Trade-off between accuracy, cost, and QoS using a beacon-on-demand strategy and Kalman filtering over a VANET. Digit. Commun. Netw. 4, 13-26 (2018). https://doi.org/10.1016/j.dcan.2017.09.001.

23. Nguyen, U.C., Tran, D.T., Nguyen, G.V.: A Taxonomy of Applying Filter Techniques to Improve the Available Bandwidth Estimations. In: Proceedings of the 8th International Conference on Ubiquitous Information Management and Communication. pp. 18:1-18:8. ACM, New York, NY, USA (2014). https://doi.org/10.1145/2557977.2558004.

24. Liu, H., Cheng, L.: Available Bandwidth Estimation Strategy Based on the Network Allocation Vector. J. Netw. 7, 2089-2095 (2012). https://doi.org/10.4304/jnw.7.12.2089-2095.

25. da Silva Mineiro, E.P., Muchaluat-Saade, D.C.: CAC-OLSR: Extending OLSR to Provide Admission Control in Wireless Mesh Networks. Int. J. Wirel. Inf. Netw. 21, 223-237 (2014). https://doi.org/10.1007/s10776-014-0242-z.

26. Gowda, G.S., Srikrishna, P.C., Dhruve, K.D.: Wireless Measurement Scheme for Bandwidth Estimation in Multihop Wireless Adhoc Network. Glob. J. Comput. Sci. Technol. 13, 11 (2013).

27. Chaudhari, S.S., Biradar, R.C.: Collision probability based Available Bandwidth estimation in Mobile Ad Hoc Networks. In: Applications of Digital Information and Web Technologies (ICADIWT), 2014 Fifth International Conference on the. pp. 244-249. , Bengaluru (2014). https://doi.org/10.1109/ICADIWT.2014.6814665.

28. Yang, T., Jin, Y., Chen, Y., Jin, Y.: RT-WABest: A novel end-to-end bandwidth estimation tool in IEEE 802.11 wireless network. Int. J. Distrib. Sens. Netw. 13, 1550147717694889 (2017). https://doi.org/10.1177/1550147717694889.

29. Manimekalai, M., Anitha, S.: Quality Of Service Routing Based On Bandwidth Estimation For Mobile Ad Hoc Networks. Int. J. Sci. Eng. Comput. Technol. 6, 195 (2016).

30. Li, J., Blake, C., Couto, D.S.J.D., Lee, H.I., Morris, R.: Capacity of Ad Hoc wireless networks. In: Proceedings of the 7th annual international conference on Mobile computing and networking. pp. 61-69. ACM, New York, NY, USA (2001). https://doi.org/10.1145/381677.381684.

31. Zhao, H., Wang, S., Xi, Y., Wei, J.: Modeling intra-flow contention problem in IEEE 802.11 wireless multi-hop networks. IEEE Commun. Lett. 14, 18-20 (2010). https://doi.org/10.1109/LCOMM.2010.01.090224.

32. Sarr, C., Chaudet, C., Chelius, G., Lassous, I.G.: A node-based available bandwidth evaluation in IEEE 802.11 ad hoc networks. Int. Conf. Parallel Distrib. Syst. 2, 68-72 (2006). https://doi.org/10.1109/ICPADS.2005.37.

33. Nyambo, B.M., Janssens, G.K., Lamote, W.: Bandwidth Estimation in Wireless Mobile Ad Hoc Networks. J. Ubiquitous Syst. Pervasive Netw. 6, 8 (2015). https://doi.org/10.5383/JUSPN.06.02.003.

34. Shah, S.H., Chen, K., Nahrstedt, K.: Dynamic bandwidth management in single-hop ad hoc wireless networks. Mob. Netw. Appl. 10, 199-217 (2005). https://doi.org/10.1023/B:MONE.0000048555.72514.9a.

35. Perkins, C., Belding-Royer, E., Das, S.: Ad hoc On-Demand Distance Vector (AODV) Routing, RFC 3561, http://tools.ietf.org/html/rfc3561, (2003).

36. de Renesse, R., Friderikos, V., Aghvami, H.: Towards Providing Adaptive Quality of Service in Mobile Ad-Hoc Networks. In: IEEE 63rd Vehicular Technology Conference. pp. 518-522. , Melbourne, Australia (2006). https://doi.org/10.1109/VETECS.2006.1682878.

37. Dai, J., Ishibashi, K., Yamao, Y.: Highly Efficient Multi-Hop Packet Transmission Using Intra-Flow Interference Cancellation and Maximal-Ratio Combining. IEEE Trans. Wirel. Commun. 14, 5998-6011 (2015). https://doi.org/10.1109/TWC.2015.2446978. 
38. Video Traces Research Group: YUV Sequences, http://trace.eas.asu.edu/yuv/index.html.

39. ITU-T: Recommendation H.265 High efficiency video coding, https://www.itu.int/rec/T-REC-H.265201802-I/en, (2018).

40. International Organization for Standardization: ISO/IEC 23009-1 Information technology — Dynamic adaptive streaming over HTTP (DASH),

https://standards.iso.org/ittf/PubliclyAvailableStandards/index.html, (2014). 